\title{
New Persistent Back-End for the ATLAS Online Information Service
}

Igor SOLOVIEV - University of California, Irvine, USA (isolov@cern.ch) Alexandru SICOE - CERN, Geneva, Switzerland

\section{Introduction}

The Trigger and Data Acquisition (TDAQ) [1] and detector systems of the ATLAS experiment deploy more than 3000 computers, running more than 15000 concurrent processes, to perform the selection, recording and monitoring of the proton collisions data in ATLAS. Most of these processes are using TDAQ Information Service (IS) system [2] to produce and share operational monitoring data for inter-process communication and analysis of the systems.

The IS has no persistence storage and only few of these data are archived by dedicated applications into offline databases. The rest of the data remained transient and lost at the end of a data taking session.

To save these data for later, offline analysis of the quality of data taking and to help investigating the behavior of the system by experts, the first prototype of a new Persistent Back-End for the Atlas Information System of TDAQ (P-BEAST) was developed and deployed in the second half of 2012.

\section{Challenges}

- Complex IS data schema is based on object data model (classes - objects attributes - types), where a type can be primitive value (string, number) or nested class, or array of them

- IS data schema can be changed in time, so support for schema evolution is needed

- Deal with huge amount of IS data: $\sim 300 \mathrm{~K}$ TDAQ IS objects resulting $\sim 5 \mathrm{M}$ independent variables (attributes of information objects) increasing database size by -30-50 GB per day during LHC Run I

- Be ready for high peaks of data updates (up to $20 \mathrm{~K}$ object updates per second) during change of data taking system state or conditions

- The IS data (callbacks) may come chronologically unordered, so need to be sorted by time

- May not introduce back pressure on IS system

- Limited resources in experiment area (Point-1) in terms of number of computers (few), their disk spaces (max 10-20 TB in total) and outgoing network bandwidth

\section{New P-BEAST (since 2013)}

Improved Cassandra-based P-BEAST on better hw can be used as intermediate buffer between IS and EOS storage, but decided to use long shutdown between LHC runs to design and implement new P-BEAST satisfying all challenging requirements. Implement custom efficient binary format with random data access inside data file based on the Google Protobuf buffer encoding [4] for data compactness, compression, high performance, cross-platform and programming language interoperability. The remote access to P-BEAST data is implemented on top of CORBA which is widely used by TDAQ for inter-process communication.

- The IS receiver gathers IS data, keeps in memory buffer, reorders \& removes duplicated data (compact) and saves to files on local storage (every $2 \mathrm{~h}$ )

- The merger combines above files after certain age ( 1 week) into a file,

compresses and copy on EOS

- The server provides interface to read

That remotely. Online data (not yet

saved) can also be read from receiver.

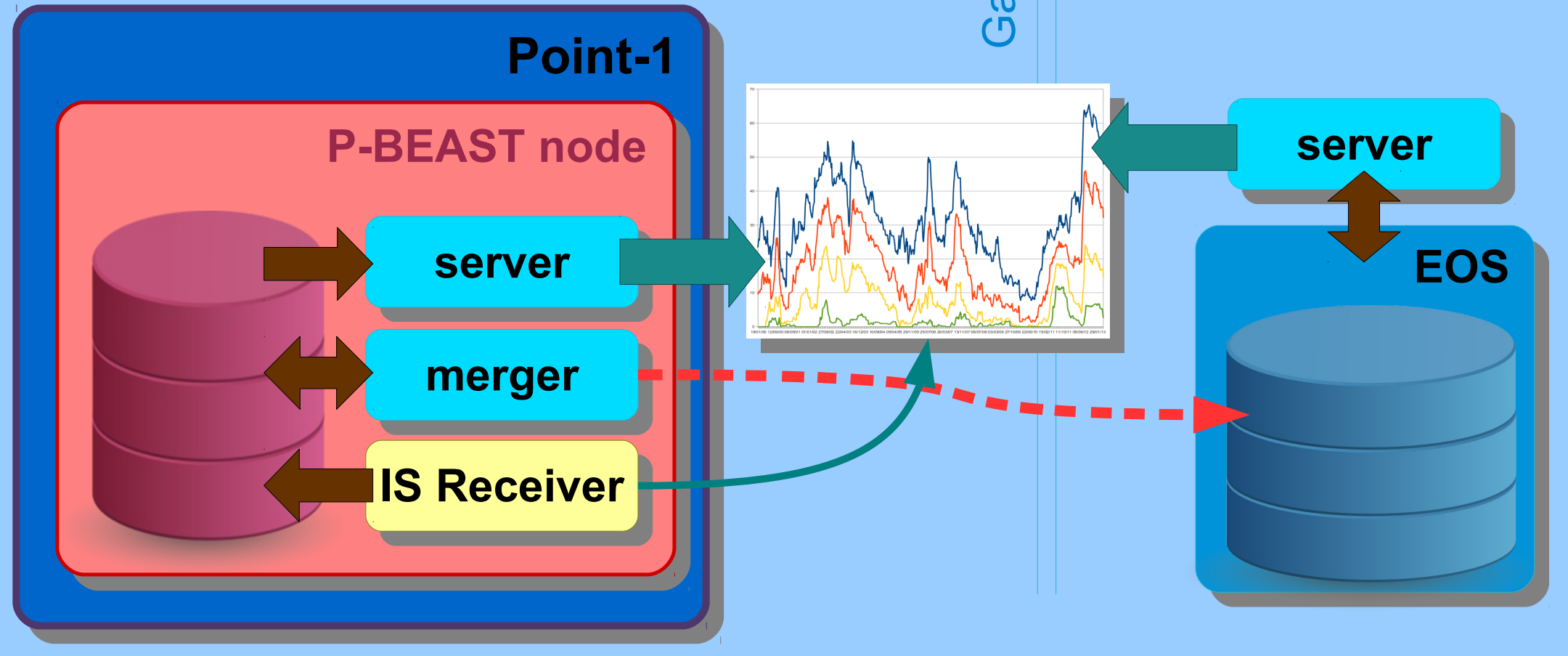

\section{Interfaces}

1. Graphical interactive Web-based monitoring data displays and dashboards:

- SPLUNK - horizontal scaling, rich querying and data transformation facilities, and sophisticated graphical Web interfaces; saves data in own DB (big latency for visualization of large amount of data imported on request). Not clear, how to remove data, when they are no more needed. - Use lightweight in-memory database, import data on user request, perform required data transformations (down-sampling, aggregations) and render plots on client side using some dynamic chart library. The Redis in-memory DB is successfully used in prototype together with Dygraphs JavaScript chart library (see picture)

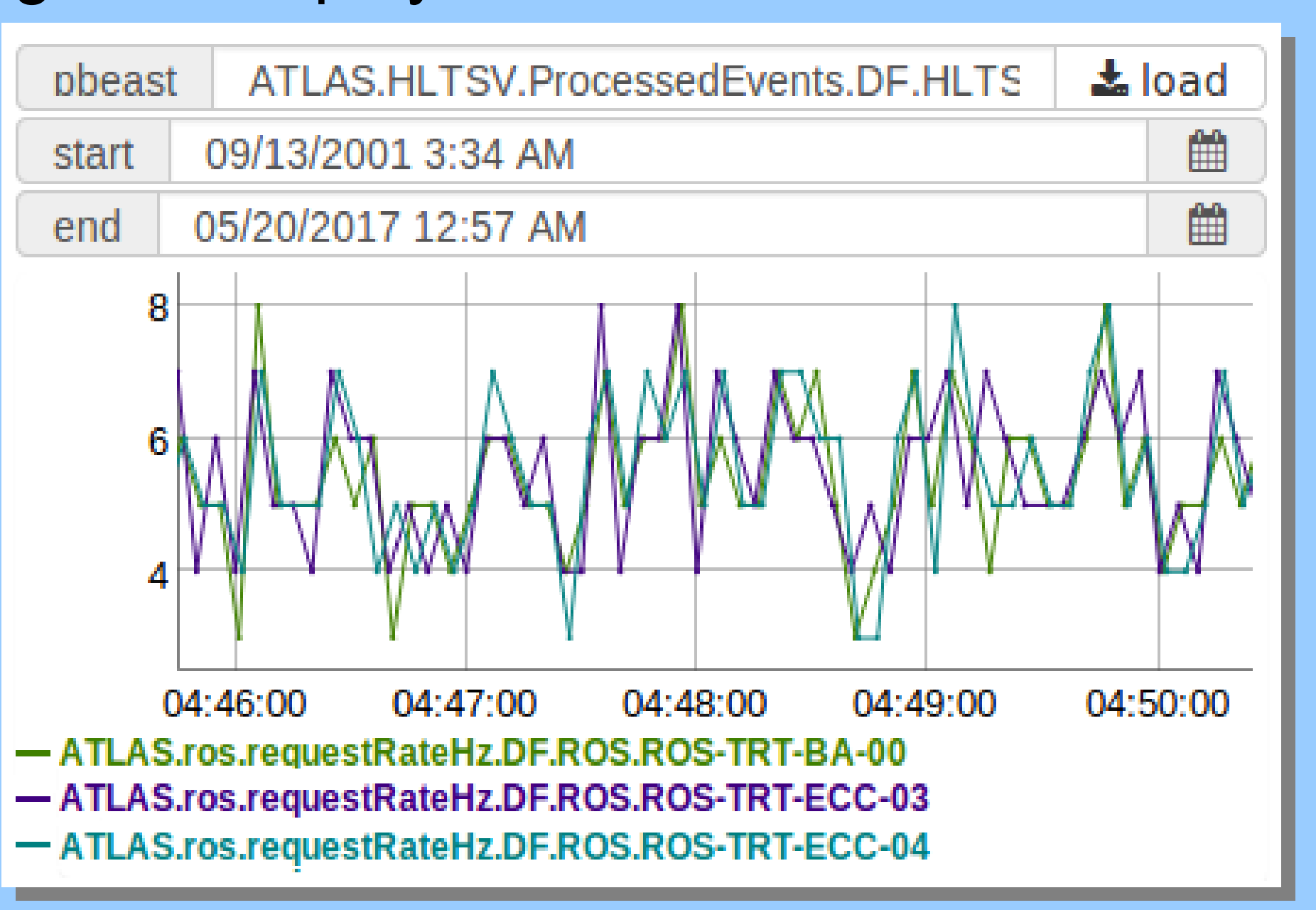

2. Reconstruct IS objects offline and inject to the TDAQ expert system for its verification (simulating real data taking run conditions).

\section{P-BEAST Requirements}

- Configurable to select which IS data to archive

- Gather IS data, process and save to database

- Provide interface to retrieve data

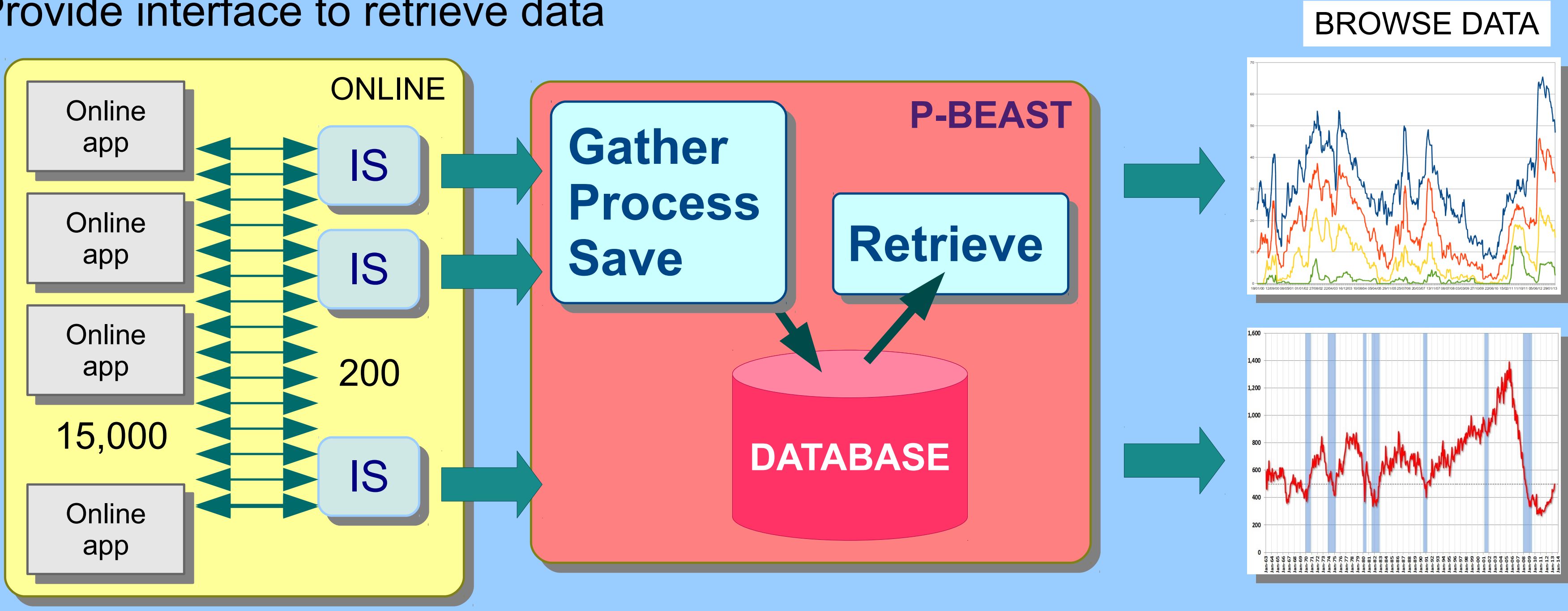

\section{Cassandra P-BEAST Implementation (2012-2013)}

After comprehensive evaluation during 2012 implemented first P-BEAST [3] using Java-based Cassandra NoSQL DB (schema flexibility, horizontal scalability, high performance). Deployed the DB on 3 powerful nodes on Point-1 with DB replication factor two, created IS injectors and moved aged data on EOS as compressed JSON files. No Cassandra DB problems with this deployment model. However

- Data model is not fully compatible

(forced to duplicate some data)

- Cassandra DB overhead

- Complicated procedure to remove old data (compaction) does not allow to use more than $40 \%$ of disk and data replication factor of 2 result only $2 \frac{1}{4}$ $\mathrm{GB}$ of $12 \mathrm{~GB}$ are available for data

- Implemented support for primitive IS types only, ignore IS objects deletion, ignore time unordering of IS callbacks - Had performance problems at high - Had performance problems at high

- No good solution to read EOS data
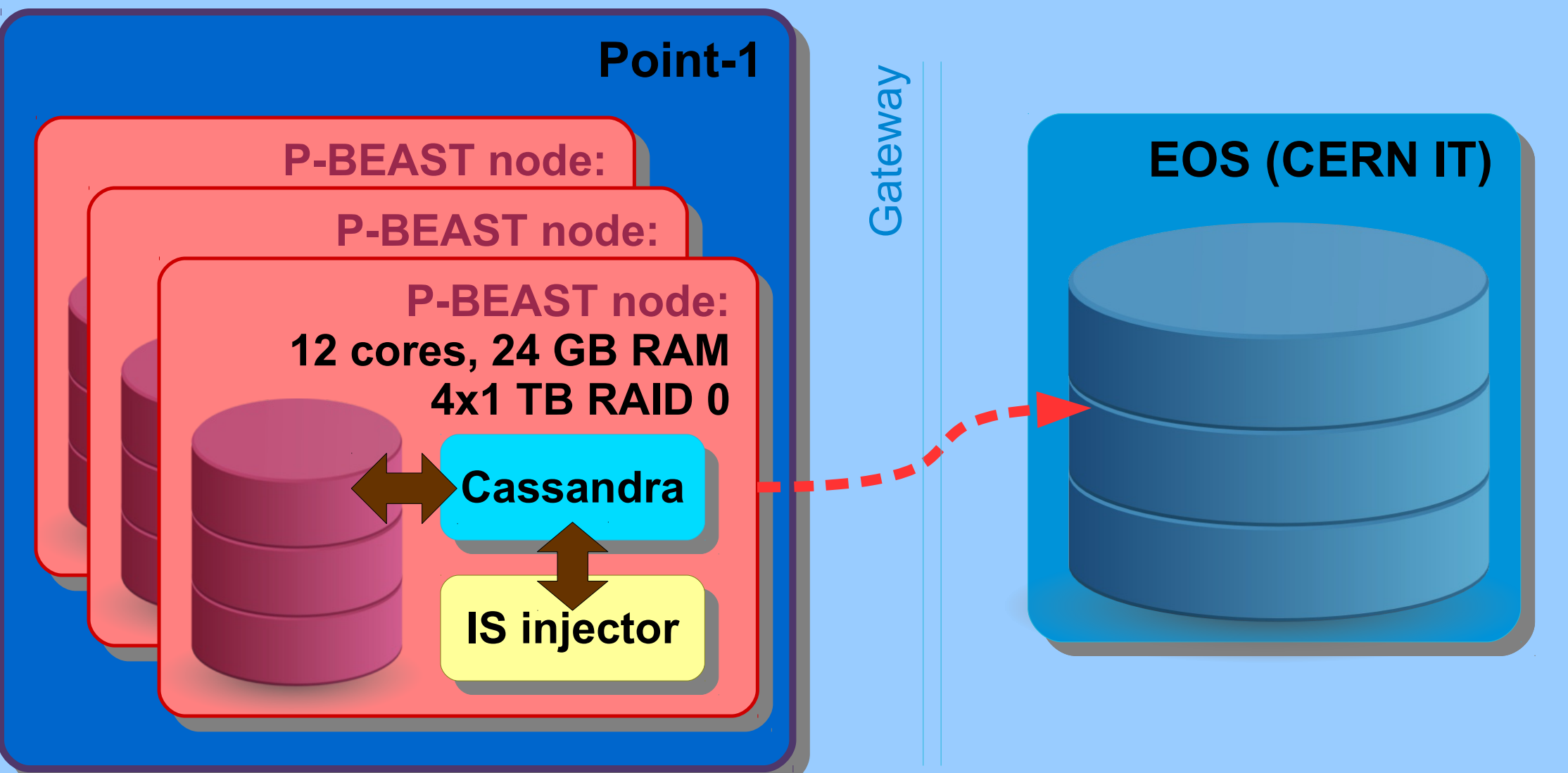

\section{Integration and Performance Tests}

The new C++-based P-BEAST service was tested during TDAQ technical and ATLAS milestone runs. The main emphasis was to test performance of the receiver in environment close to future LHC Run 2. The graph presents accepted rates for single P-BEAST receiver for object updates (i.e. number of IS callbacks per second) and tota number of their attributes (i.e. number of inserted data points). No IS non-responsive client problems occurred during these tests.

- The tests were performed on a node

previously used by Cassandra DB. The

only difference, that the RAID disk array was reconfigured to level 5 , since there is no more replication of data across different nodes as it was with Cassandra.

If necessary, the design allows to use more than one P-BEAST receiver pe TDAQ partition, if performance will be insufficient. At present a single node is capable to sustain two times higher rates, than 3 nodes running Cassandra.

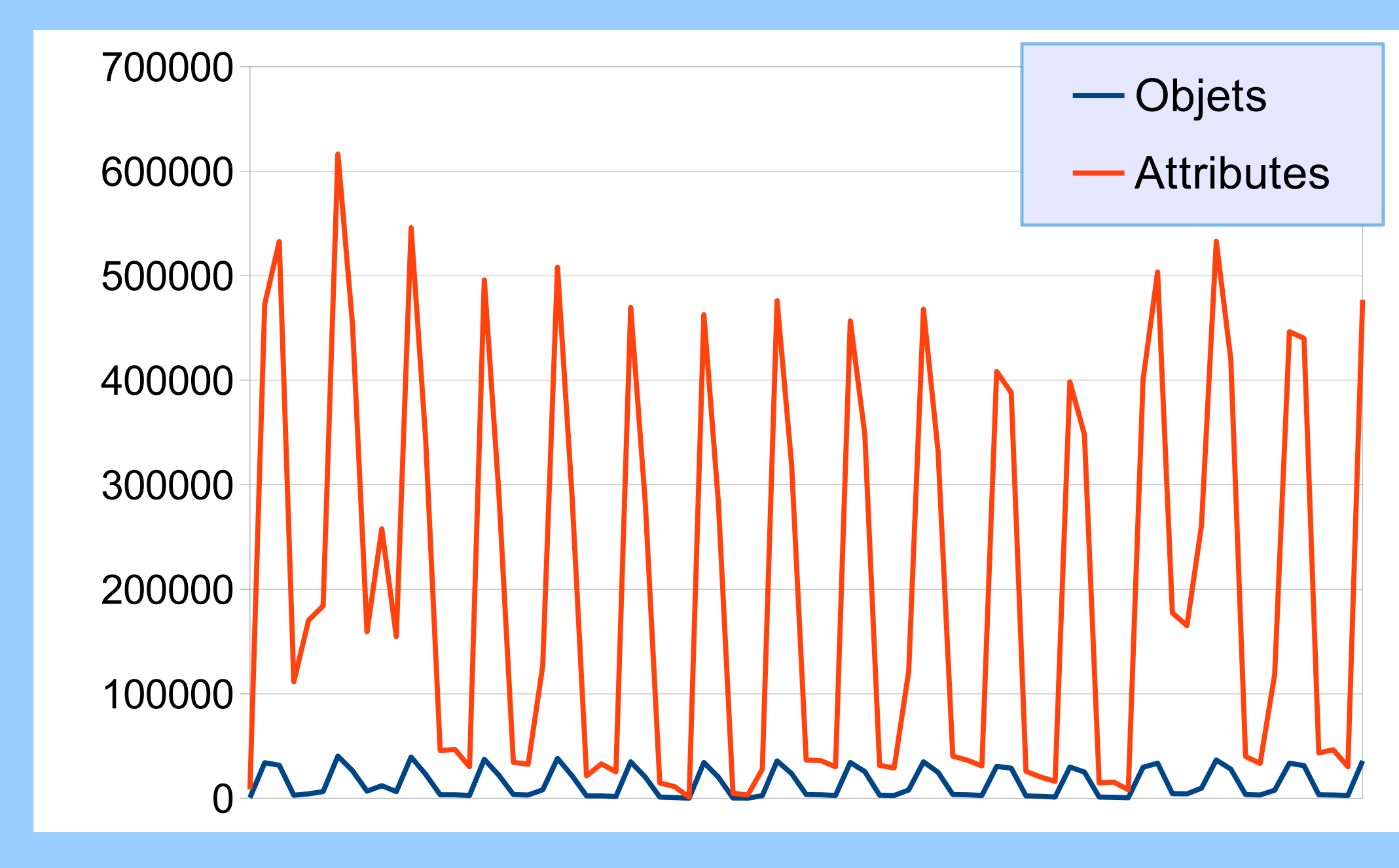

\section{Conclusions}

By results of the tests performed during TDAQ technical and ATLAS milestone runs the new P-BEAST service is able to gather and to save IS monitoring data at conditions similar to production environment without loosing any piece of the data using a single computer. Nevertheless the design of the service allows running multiple instances for horizontal scalability if needed. The file format based on Google protocol buffer encoding allows to efficiently compact numeric values and to compress data with the zip algorithm keeping random access to data point series by object names. Thus the gathering and storage functionality of new P-BEAST has been validated. The architecture of the service allows to store and to access data using repositories located inside Point-1 and external EOS service. The CORBA interface allows to access data using different programming languages and running on computers which have no direct access to the files stored on P-BEAST repositories. There are prototypes for Web-based graphical browsers and dashboards of the P-BEAST data. There are no known problems with this approach and ongoing development should result appearance of fully functional graphical backend at short timescale.

\section{References}

[1] - ATLAS Collaboration, "Atlas high-level trigger, data acquisition and controls technical design report", http://cdsweb.cern.ch/record/616089, 2003

[2] - S. Kolos, G. Boutsioukis, R. Hauser, "High-Performance Scalable Information Service for the ATLAS Experiment", 2012 J. Phys.: Conf. Ser. 396012026

[3] - A. Sicoe, et al., "A persistent back-end for the ATLAS TDAQ online information service (P-BEAST)", 2012, J. Phys.: Conf. Ser. 368012002

[4] - "Protocol Buffers", https://developers.google.com/protocol-buffers/ 